\title{
Bacteriological Profile from Nasopharyngeal Aspirates in Children Under 5 Years with Acute Respiratory Infections
}

\author{
Dr. Balanuta Ana-Mihaela*, Dr. Bujor Dina*, Dr. Horodisteanu-Banuh Adela*, Dr. Savoschin Dorina*, Dr. Grin \\ Olesea* $^{*}$
}

\author{
Scientific Department of Paediatrics, PHMI Institute of Mother and Child, Republic of Moldova \\ DOI: 10.29322/IJSRP.11.10.2021.p11828 \\ http://dx.doi.org/10.29322/IJSRP.11.10.2021.p11828
}

\begin{abstract}
Introduction. Potentially pathogenic bacteria that colonize the nasopharynx can cause a range of respiratory tract infections. There is a lack of related national information about the bacterial spectrum in the nasopharynx that can cause acute respiratory infections in small children. These findings expressed the necessity to enhance a study, which results can play an essential role in improving therapeutic decisions in the national pediatric respiratory care field.

Materials and methods. Nasopharyngeal samples were collected from 75 hospitalized children within the Mother and Child Institute and Municipal Clinical Children's Hospital No. 1 in Chisinau aged $\geq 8$ weeks and over $\leq 5$ years who showed symptoms of a respiratory infection. Bacterial pathogens were detected using traditional culture methods, and disc diffusion tests were used to determine antibiotic resistance.
\end{abstract}

Results. Among the 75 analyzed samples, 58 (77,33\%) were positive for bacterial infection. The most prevalent bacterial agent colonizing the nasopharynx in small children was $S$. aureus (32\%), followed by Moraxella Catharhalis (20\%), S.Dysgalactiae group C (20\%). Regarding vaccine preventable bacteria S. pneumoniae was noted in $1.33 \%$ of cases and $\mathrm{H}$. Influenzae in 4\%. Resistance levels to penicillins, carbapenems, macrolides, Sulfamethoxazole-Trimethoprim varied in different bacteria.

Conclusion. Microbial distribution and their patterns of antibiotic susceptibility revealed alarming data due to the rational use of antibiotics in acute respiratory infections in children under 5 years. Thus, further results from this national study should be considered for empirical decisions during antibiotic therapy for Moldavian pediatric patients.

Index Terms- antibiotic resistance, bacteria, nasopharynx, pediatric, respiratory tract.

\section{INTRODUCTION}

$\mathrm{B}$ uilding on the work of others, nasopharyngeal microbiota has age-dependent dissimilarities. Thereby, the nasopharynx of small children is more colonized than in adults [1]. Representative data showed that children under 5 years are more often colonized by pneumococci than older children $[1,2]$. On a related note, potentially pathogenic bacteria that colonize the nasopharynx can cause a range of respiratory tract infections [3, 4]. It is common knowledge that Acute respiratory tract infection (ARI) is widely recognized as the leading cause of mortality and morbidity in children under five years, especially in developing countries $[5,6]$. According to the latest statistical data in our country, the incidence of respiratory diseases in children under 5 years, per 1000 children of the respective age, in 2019, was 905,3 new cases [7]. There is a lack of related national information about the bacterial spectrum in the nasopharynx and antibiotic resistance phenomenon of the most common microorganisms that can cause acute respiratory infections in small children. These findings expressed the necessity to enhance a study, which results can play an essential role in helping to develop and implement national antibiotic use policies in the national pediatric respiratory care field.

\section{MATERIALS AND METHODS}

This study is currently underway. It is being carried out within the National project "The impact of immunization on the morbidity and mortality of children with respiratory diseases in the Republic of Moldova" (project code) - 20.80009.8007.08. Participation in the study was entirely voluntary, expressed by written consent, obtained from each of the participants included in the study. Between December 2020 and June 2021, nasopharyngeal samples were collected from 75 hospitalized children within the Mother and Child Institute and Municipal Clinical Children's Hospital No. 1 in Chisinau aged $\geq 8$ weeks and over $\leq 5$ years who showed symptoms of a respiratory infection (figure 1). Nasopharyngeal samples were obtained before commencing antibiotic therapy. Bacterial pathogens were detected using traditional culture methods, and disc diffusion tests were used to determine antibiotic resistance. Also testing of strains isolated from antibiotics and interpretation of results obtained according to the requirements of the EUCAST standard. Descriptive statistics was performed using Microsoft excel spreadsheet 2010. Categorical data was described as percentages. 


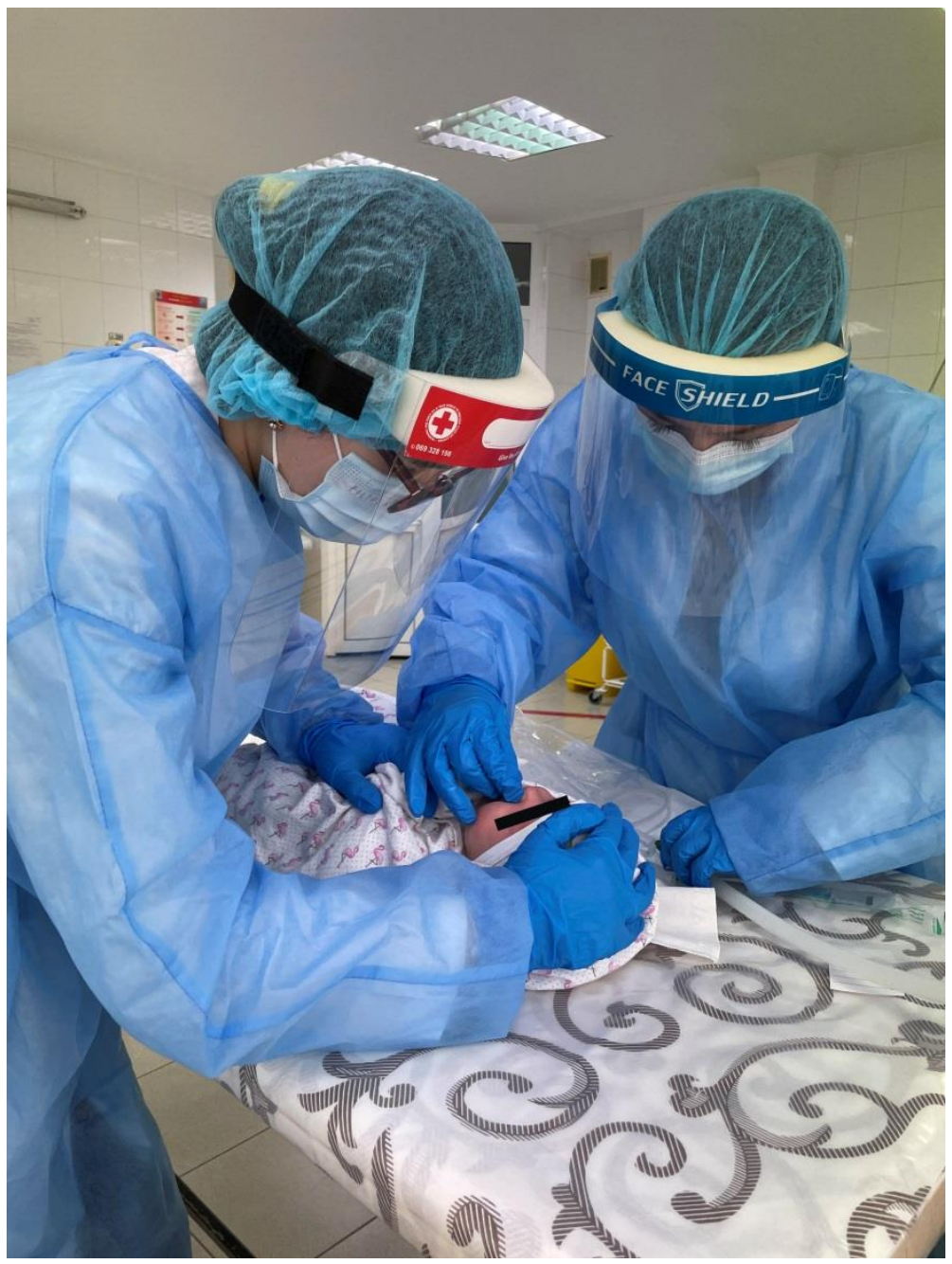

Figure 1: Nasopharyngeal aspiration in an infant with acute respiratory symptoms.

\section{RESULTS}

The mean age at presentation was $18 \pm 1.6$ years, the median age was about 14 moths. Out of 75 patients there were $54.66 \%$ of boys, with Boy / girl ratio $=1.20 / 1$. There was an uneven territorial distribution of cases, thereby in urban areas $92 \%$, and in rural areas $8 \%$; urban / rural residence ratio $=11.5 / 1$. The level of family income in $69 \%$ of cases was declared as average. The analysis of anamnestic data revealed that about $61.33 \%$ of these children were born at term and about $58.66 \%$ had a birth weight of 2500-3499 g; prematurity being noticed in $10 \%$ of children. The assessment of vaccine status revealed $9.33 \%$ of unvaccinated children. The evaluation of anthropometric indices found a BMI corresponding to the normal weight percentile in $74.68 \%$ of cases, underweight in $9.33 \%$ and obesity in $6.66 \%$ of cases. About $16 \%$ of children frequently undergo ARI, thus $33.33 \%$ have been hospitalized during the last 12 months. Pneumonia was diagnosed at the time of hospitalization in $21.33 \%$ of cases. Among the 75 analyzed samples, 58 (77,33\%) were positive for bacterial infection. Bacteriological examination of nasopharyngeal specimen isolates identified $S$. aureus as a predominant bacterial agent colonizing the nasopharynx in young children (32\%), being resistant in $66 \%$ to penicillins, in $12 \%$ to macrolides and in 20\% with a high sensitivity to antibiotics. Moraxella Catharhalis was highlighted in $20 \%$ of cases, the profile of antimicrobial resistance outlined in $40 \%$ of cases resistance to meropenem. Likewise in $20 \%$ of cases, S.Dysgalactiae group C and G were detected, in $26 \%$ resistant to penicillins, in $27 \%$ resistant to SulfamethoxazoleTrimethoprim and in 20\% with an increased sensitivity to all groups of antibiotics. The spectrum of respiratory etiology preventable by vaccination noted $S$. pneumoniae in $1.33 \%$ of cases and $H$. Influenzae in $4 \%$, at the same time they were noted in small titers, which suggests the carrier status highlighting a potential risk of developing invasive infections. It is noteworthy that $8 \%$ of the identified microorganisms showed extremely resistant bacteria such as $S$. Aureus MRSA, Burkholderia cepacia, Acinetobacter lwoffii. At the same time it is important to mention that in the case of 10 collected specimens (13.3\% of cases) were highlighted mixed bacterial growths.

\section{DISCUSSION}

This study has limitations. Presented data are not sufficient yet, to allow overall conclusions, to be drawn, hence the need to 
continue this study with many more patients. On the other hand, COVID-19 pandemic caused a huge decrease in pediatric admissions to the hospital. Also, a lot of patients had already started the antibiotic therapy before the admission, which matched them as ineligible to be included in this study.

To begin with, there are limited studies focused on investigating nasopharyngeal carriage during disease episodes, our data provided interesting findings about prevalent bacterial agents in the nasopharyngeal aspirate of our pediatric patients and elucidated alarming facts due to antibiotic resistance phenomenon. Furthermore we have reported a slight incidence of Haemophilus influenzae, and Streptococcus pneumoniae, mainly those data reflect vaccine polices in our country. Whereas the carriage rates of $S$. pneumoniae and Haemophilus influenza were minor, that of Staphylococcus aureus, showed, in our study, an opposite tendency. According to recent studies $[8,9,10]$ it has been ascertained a continued decrease in the overall pneumococcal carriage, mainly fuelled by the decline in vaccine serotypes after vaccine introduction in several countries. Studying the susceptibility profile revealed an worrying prevalence and resistance pattern of Moraxella Catharhalis to meropenem. As long as worldwide are seen, high resistance rates to many firstline antibiotics [11], this finding was unexpected.

\section{CONCLUSION}

Microbial distribution and their patterns of antibiotic susceptibility revealed alarming data due to the rational use of antibiotics in acute respiratory infections in children under 5 years. Thus, further results from this national study should be considered for empirical decisions during antibiotic therapy for Moldavian pediatric patients.

\section{REFERENCES}

[1] Salter SJ, Turner C, Watthanaworawit W, de Goffau MC, Wagner J, et al. (2017) A longitudinal study of the infant nasopharyngeal microbiota: The effects of age, illness and antibiotic use in a cohort of South East Asian children. PLOS Neglected Tropical Diseases 11(10): e0005975. https://doi.org/10.1371/journal.pntd.0005975

[2] Jonathan Reisman, Karen Rudolph, Dana Bruden, Debby Hurlburt, Michael G. Bruce, Thomas Hennessy, Risk Factors for Pneumococcal Colonization of the Nasopharynx in Alaska Native Adults and Children, Journal of the Pediatric Infectious Diseases Society, Volume 3, Issue 2, June 2014, Pages 104-111, https://doi.org/10.1093/jpids/pit069

[3] Diagne, G. , Kane, A. , Mbaye, A. , Coundoul, A. , Sow, S. , Bop, K. , Sow, A. , Ba, I. and Ndiaye, O. (2021) Bacteriological Profile of Acute Respiratory Infections in Children: About a Prospective Study at the Albert Royer Hospital in Dakar. Open Journal of Pediatrics, 11, 71-77. doi: 10.4236/ojped.2021.111007

[4] Stearns JC, Davidson CJ, McKeon S, Whelan FJ, Fontes ME, Schryvers AB, et al. Culture and molecular-based profiles show shifts in bacterial communities of the upper respiratory tract that occur with age. ISME Journal. 2015;9(5):1246-59. pmid:25575312.
[5] Alamsyahi A, Kurniya T, Ikhtiaruddin I, Rasyid Z. Determinants of Acute Respiratory Infections Incidence in Children Under Five in the Working Area of the Siak Hulu II Community Health Center in Kampar Regency. Open Access Maced J Med Sci [Internet]. 2021 Jan. 24 [cited 2021 Aug. 16];9(E):59-63.

[6] Rudan, Igor et al. "Global estimate of the incidence of clinical pneumonia among children under five years of age." Bulletin of the World Health Organization vol. 82,12 (2004): 895-903.

[7] Cara O, Spataru A, Cojocari N et al. Children of Moldova Statistical Publication. National Bureau of Statistics of the Republic of Moldova. Unicef. 2020.

[8] Løvlie A, Vestrheim DF, Aaberge IS, Steens A. Changes in pneumococcal carriage prevalence and factors associated with carriage in Norwegian children, four years after introduction of PCV13. BMC Infect Dis. 2020 Jan 10;20(1):29. doi: 10.1186/s12879-019-4754-0. PMID: 31924177; PMCID: PMC6954625.

[9] Alfayate Miguélez S, Yague Guirao G, Menasalvas Ruíz AI et al. Impact of Pneumococcal Vaccination in the Nasopharyngeal Carriage of Streptococcus pneumoniae in Healthy Children of the Murcia Region in Spain. Vaccines (Basel). 2020 Dec 28;9(1):14. doi: 10.3390/vaccines9010014. PMID: 33379235; PMCID: PMC7823743.

[10] Tin Tin Htar M, van Den Biggelaar AHJ, Sings H, Ferreira G et al. The impact of routine childhood immunization with higher-valent pneumococcal conjugate vaccines on antimicrobial-resistant pneumococcal diseases and carriage: a systematic literature review. Expert Rev Vaccines. 2019 Oct; 18(10):1069-1089. doi: 10.1080/14760584.2019.1676155. Epub 2019 Oct 22. PMID: 31585049.

[11] Mhondoro, M., Ndlovu, N., Bangure, D. et al. Trends in antimicrobial resistance of bacterial pathogens in Harare, Zimbabwe, 2012-2017: a secondary dataset analysis. BMC Infect Dis 19, 746 (2019). https://doi.org/10.1186/s12879-019-4295-6

\section{AUTHORS}

First Author - Dr. Balanuta Ana-Mihaela, Scientific Department of Paediatrics, PHMI Institute of Mother and Child, Republic of Moldova

Second Author - Dr. Bujor Dina, Scientific Department of Paediatrics, PHMI Institute of Mother and Child, Republic of Moldova

Third Author - Dr. Horodisteanu-Banuh Adela, Scientific Department of Paediatrics, PHMI Institute of Mother and Child, Republic of Moldova

Fourth Author - Dr. Savoschin Dorina, Scientific Department of Paediatrics, PHMI Institute of Mother and Child, Republic of Moldova

Fourth Author - Dr. Grin Olesea, Scientific Department of Paediatrics, PHMI Institute of Mother and Child, Republic of Moldova

Corresponding Author: Dr. Revenco

Ninel,ninel.revenco@usmf.md,MD,PhD, Doctor Habilitatus in Medicine, Professor, Chief of Department of Pediatrics,State University of Medicine and Pharmacy "Nicolae Testemitanu", Chisinau, Republic of Moldova; 